\title{
Sucrose and cardiovascular disease
}

\section{By J. Yudkin, Queen Elizabeth College, London W8 7 AII}

Like other speakers today, I propose to confine myself to one manifestation of cardiovascular disease, namely ischaemic heart disease (IHD). It is unlikely that there is any single statement one could make about cardiovascular disease in general, or about IHD in particular, that would command universal agreement. The natural history of atherosclerosis, the relationship between atherosclerosis and IHD, the relative prevalence of IHD in different countries, whether there has been a real increase in its prevalence in affluent countries over the past century and if so the size of this increase: these are only a few of the questions about which debate continues. I believe however that there would be widespread if not universal agreement about the following three statements.

First, there are several environmental factors that may produce IHD; among those that have been suggested are cigarette smoking, physical inactivity, stress, and diet.

Second, persons suffering from IHD show a wide range of abnormalities, of which one is the common though not universal high level of plasma cholesterol.

Third, IHD is associated in varying degree with other diseases such as diabetes, peripheral vascular disease, and peptic ulceration.

With these points in mind, let me then summarize the evidence that dietary sucrose is a factor in the aetiology of IHD.

\section{Epidemiology}

Epidemiology is a potent weapon for identifying clues as to aetiology, but it cannot of itself provide proof. One limitation is that several factors that may be of aetiological significance tend to occur together. There is a general association in different populations between the prevalence of IHD and affluence; as a result, there is an association between disease prevalence and any one of several indices of affluence, including electricity consumption, motor cars, radio and television, physical inactivity, tobacco consumption, and the dietary intake of energy and of items such as animal protein, fat, sucrose, and vitamin $B_{12}$.

On the basis of epidemiology alone, any one of these factors might be considered to be of aetiological significance, though clearly some are more plausible than are others. It is possible though not very likely that the possession of a television set in itself predisposes to the development of IHD, and that the smoking of cigarettes is an irrelevant concomitant.

A second limitation of epidemiological studies derives from the fact that there is 
indeed not one but several environmental factors involved in the production of IHD. We cannot therefore expect that any one isolated factor will show an exact association with the disease, in either horizontal or longitudinal epidemiological studies. There is for example a good association from interpopulation studies between the prevalence of the disease and the consumption of fat, or of sucrose. I do not however feel we shall make any more progress by attempting to assess whether the association with the one dietary component is better than the association with the other, since we are far from knowing what are all the other factors that may contribute to the disease in each of the countries, or what is the relative size of these factors. Epidemiological studies with populations have served their purpose in providing clues for possible aetiological factors; having discovered these clues, we now need to search for evidence from other sources to see whether they lead us to solutions or to dead ends.

Epidemiological studies can also be made with individuals, where one attempts to characterize those who do develop the disease and those who do not. The only environmental factor that has been unequivocally shown both in prospective and retrospective studies to be associated with IHD is cigarette smoking. On the other hand, no difference has ever been demonstrated in the amounts or kinds of dietary fat eaten by persons with or without the disease. Between these two extremes lies the situation with sucrose, in which some observers have found that persons who developed IHD have been eating more sucrose than have those without the disease, but some observers have not. This however cannot be taken as proof that we may absolve sucrose as a factor in causing the disease. We may remind ourselves that studies in individuals on the relationship between obesity and food intake find either only a slight relationship or none at all. No one would take this as demonstrating that food intake has nothing to do with the development of obesity.

\section{Experimental evidence}

Having derived hypotheses from clues provided by epidemiological evidence, we now proceed to test them against evidence provided by experiment. The experiments can be made with human volunteers or with laboratory animals. Clearly we shall not attempt in man to produce IHD; on the other hand, in laboratory animals, no one has produced an exact replica of the human disease. We have therefore to look for one or more concomitants of the disease. The most obvious is atherosclerosis; others are raised concentrations of plasma cholesterol and plasma triglyceride, an increased platelet adhesiveness, an abnormal pattern of platelet electrophoretic mobility in the presence of ADP, an increased concentration of plasma insulin, and a diminished glucose tolerance. Not all of these are found in every individual with IHD; on the other hand it is becoming increasingly evident that the feature most commonly assessed - the concentration of plasma cholesterol - is not the one that is most frequently altered in IHD. Ideally, experiments should seek to determine whether a suspected aetiological factor produces all or most of the changes found in the disease. Again ideally, evidence should be sought from several species of laboratory animals as well as from human subjects, since there are con- 
siderable differences between species in their metabolic response to particular changes in diet or in other factors.

This is the approach that we have adopted in seeking evidence to support the hypothesis that sucrose is one of the causative agents in IHD. We have sought to measure the effects of dietary sucrose upon several bases both in man and in two or three species of laboratory animals. Since the particular hypothesis that we are testing is that one of the causes of IHD is the replacement of part of the starch in our diet by sucrose, our experiments have been based on a comparison of the effects of diets containing starch and little or no sucrose with those of diets in which all or part of the starch is replaced by an equivalent amount of sucrose.

We have measured the customary dietary intake of young men and then added some $200 \mathrm{~g}$ of sucrose at the expense of the same amount of starch. After 2 weeks, we have found a small but significant increase in the concentration of plasma triglyceride in all of the subjects; in some experiments we have also found a smaller but still significant increase in the concentration of plasma cholesterol, and an improvement in glucose tolerance (Yudkin and Szanto, in preparation). Cohen, Teitelbaum, Balogh \& Groen ( 1966 ), in Jerusalem, have shown in human subjects that, after several weeks, dietary sucrose produces a deterioration in glucose tolerance. In each of our experiments, other changes were seen in only a proportion of the volunteers. About $30 \%$ of them showed an increase in body-weight of about $2 \mathrm{~kg}$ after 2 weeks on the high-sucrose diet, an increase in platelet adhesiveness, a paradoxical change in motility of the platelets in the presence of ADP, and an increase in the concentrations of plasma insulin and plasma $\mathrm{I}$ I-hydroxycorticosteroid.

We have suggested that the persons in whom sucrose produces 'hyperinsulinism' are also those who are susceptible to the action of sucrose in producing IHD (Szanto \& Yudkin, 1969). One reason for this belief is that we are impressed by the evidence summarized by Mahler (I965) and by Stout \& Vallance-Owen (r969) that a high concentration of insulin may be the mechanism that produces atherosclerosis. A second reason is that an hormonal imbalance, perhaps of insulin or perhaps of an adrenocortical hormone, is a more plausible explanation of the multiple disturbances that are associated with IHD than is an explanation based on a simple biochemical aberration such as in the rate of cholesterol synthesis. The best evidence that hormonal activity is involved in IHD is the relative immunity of women to the disease, but only before the menopause.

Animal experiments have shown that sucrose induces many changes, including changes in insulin concentration. In rats, diets with sucrose given for ro weeks produce a fall in insulin concentration (Bruckdorfer, Khan \& Yudkin, 1972a); in pigs, a similar diet produces a rise in insulin concentration. Sucrose induces a rise in plasma lipid concentrations, although the particular lipids affected vary with the species. In rats, there is usually a rise in the concentration of plasma triglyceride, occasionally of phospholipid, and sometimes of cholesterol (Al-Nagdy, Miller \& Yudkin, 1970; Bruckdorfer, Khan \& Yudkin, I97I). In pigs, we have found a transitory rise in triglycerides only; in cockerels, there was a considerable rise in cholesterol only. 
Sucrose produces an enlargement of the liver, with an increase in fat content (Bruckdorfer, Khan \& Yudkin, 1972a). In cockerels, the increase in liver weight may be as much as $50 \%$. We have recently shown in the rat that the increase in liver weight is accompanied by an increase both in cell numbers and in cell size; that is sucrose induces hyperplasia as well as hypertrophy of the liver (Bender, Damji, Khan, Khan, McGregor \& Yudkin, 1972). Other notable changes in organs are a decrease in the weight of the pancreas in the pig, an increase in the weight of the kidney in the rat, and an accelerated maturation of the adrenal glands in the young rat.

Sucrose changes the activities of several enzymes involved in the metabolism of fat and of carbohydrate. In the rat, there is an increase in the activity of fatty acid synthetase, glucose-6-phosphate dehydrogenase (EC I.I.I.49), and pyruvate kinase (EC 2.7.r.40) in the liver, and a decrease in fatty acid synthetase in adipose tissue. The increase in fatty acid synthetase activity in the liver and of triglyceride in the plasma occur within $\mathrm{I} 8 \mathrm{~h}$; they fall almost as rapidly when sucrose is removed from the diet. It would be interesting to know whether the changes in enzyme activity affect the level of hormones and thus are the first link in the chain of events leading from dietary sucrose to IHD.

Other changes that occur with dietary sucrose are more clearly related to its possible role in producing arterial disease. In rats it leads to an increase in platelet aggregation. Unlike Chevillard and his collaborators (Chevillard, Portet, Combescot \& Senault-Bournique, 1968), we have not been able to produce aortic atheroma in rats with sucrose; we have, however, shown that sucrose produced an increase in the concentration of lipid in the aortic wall of the rat (Bruckdorfer, Khan \& Yudkin, I $972 b$ ). In cockerels, we have been able to produce aortic atheroma with sucrose, the area of atheroma being in direct proportion to the raised level of plasma cholesterol.

Not all the changes induced by sucrose are of direct and obvious relevance to the problem of IHD; they do nevertheless serve two important purposes. First, they help to remove one obstacle to the acceptance of the view that sucrose might be involved in the causation of a complex disease like IHD, an obstacle based on the mistaken view that sucrose is simply one of the dietary carbohydrates that can with impunity be exchanged for any other.

The second purpose served by a broad study of the effects of dietary sucrose is that some of these might be relevant to the possible role of sucrose in other diseases that are often associated with IHD. If it can be shown that sucrose is a likely cause of a disease that is known to be common in people with IHD, this would provide additional evidence, indirect but nevertheless useful, of the possible role of sucrose in IHD.

\section{Relationship of IHD with other diseases}

There is a tendency for IHD to be more common in persons suffering from several other conditions than it is in healthy persons. The relationship varies from 
being very close, as with maturity onset diabetes, to being slight or even dubious, as with obesity or gout.

It is widely agreed that middle-aged diabetics are appreciably more prone to IHD than are non-diabetics; conversely, persons with manifest atherosclerotic disease are likely to have an impaired glucose tolerance, if not more obvious signs of diabetes. This association between the two diseases has implications for our discussion of the possible aetiological role of sucrose in IHD. Firstly, as we have seen, dietary sucrose results in a diminished glucose tolerance in man and in the rat. Secondly, there is increasing evidence that maturity onset diabetes begins with a raised concentration of plasma insulin (Jackson, Mieghem \& Keller, 1972) and we have seen that this is a feature of a high-sucrose intake in a proportion of human subjects.

Though less well documented, peptic ulceration is another disease that is associated with IHD. We believe that two of our recent investigations support the view that sucrose might play a role in the causation of peptic ulceration. In the first, we conducted a trial with forty-one patients with chronic dyspepsia, of whom at least ten were suffering from peptic ulceration (Yudkin, Evans \& Smith, 1972). We were able to show that many more patients were improved by a diet low in carbohydrate, and particularly low in sugar, than were improved by the conventional sort of 'gastric' diet. In a second experiment, we showed that a high-sucrose diet for 2 weeks induced an increase of about $20 \%$ in gastric acidity, and an increase of $200 \%$ in peptic activity.

The relationship between obesity and IHD is tenuous. I believe that this is not unexpected in the light of recent research. Firstly, we have shown in young students that overeating in general, whether or not the excess was largely sucrose, produced an increase in plasma insulin in all subjects. Secondly, this overeating did not lead to anything like the degree of weight gain that would be expected from conventional teaching; as has now been demonstrated several times by my colleagues, young people tend to react to excessive energy intake by increasing their metabolic rate (Miller \& Mumford, 1967). If a raised concentration of insulin predisposes to IHD, we would expect that people who have and can maintain this ability to overeat without developing obesity would nevertheless become likely candidates for IHD because of the effect of overeating on plasma insulin. The existence of such a category however would clearly diminish the relationship between obesity and IHD.

Persons with gout seem to have a rather greater than normal risk of developing IHD, whereas subjects with atherosclerotic disease seem to have a higher than average concentration of serum uric acid. We have found that persons with gout tend to have a rather higher sucrose intake than do matched control subjects. We have not been able to show an increase in serum uric acid in our human volunteers taking a high-sucrose diet; this may have been because the experiments lasted only 2 or 3 weeks. It has however been shown that fructose raises the concentration of serum uric acid (Perheentupa \& Raivio, 1967).

\section{Conclusion}

I have dealt with three sorts of evidence that can help us in our attempts to expose 
the causes of IHD. They are epidemiology, experiment, and the association of IHD with other diseases.

When seeking evidence from epidemiology, we must bear in mind that IHD has several causes, so that epidemiology can mislead if we expect it to do more than provide clues as to aetiology.

When seeking evidence from experiment, we must bear in mind that IHD has several manifestations, so that experiment can mislead if we try to reproduce only one characteristic of IHD.

When seeking evidence from associated diseases, we must bear in mind that these too may have several causes, not all of which coincide with the causes of IHD. Our investigations can therefore mislead if we expect the associations to be universal and inevitable.

We would do well to bear these points in mind, and the simple diagram (Fig. I) was designed to help us to do this.

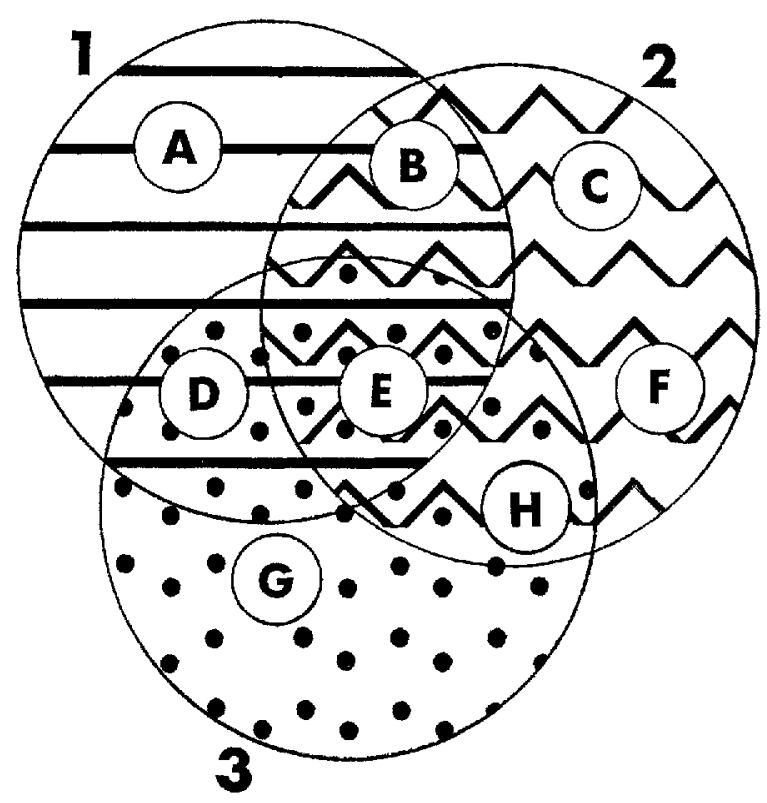

Fig. I. Association of diseases with many causes. Disease I may be caused by factors A, B, D or E; disease $\mathrm{z}$ by factors $\mathrm{B}, \mathrm{C}, \mathrm{E}, \mathrm{F}$ or $\mathrm{H}$; disease 3 by factors D, E, G or H. Factor A can cause only disease I, factor $\mathrm{C}$ or $\mathrm{F}$ only disease 2 , factor $\mathrm{G}$ only disease 3. Factor $\mathrm{B}$ can cause either disease $\mathbf{x}$ or disease 2, factor $\mathrm{D}$ either disease $\mathrm{I}$ or disease 3 , factor $\mathrm{H}$ either disease 2 or disease 3. Factor $\mathrm{E}$ can cause either disease $x$ or disease 2 or disease 3 .

\section{REFERENCES}

Al-Nagdy, S., Miller, D. S. \& Yudkin, J. (1970). Nutr. Metab. 12, 193.

Bender, A. E., Damji, K. B., Khan, M. A., Khan, I. H., McGregor, L. \& Yudkin, J. (1972). Nature, Lond. $\mathbf{2 3} 8,46 \mathrm{r}$.

Bruckdorfer, K. R., Khan, I. H. \& Yudkin, J. (1971). Nutr. Metab. 13, 36.

Bruckdorfer, K. R., Khan, I. H. \& Yudkin, J. (1972a). Biochem. f. 129, 439.

Bruckdorfer, K. R., Khan, I. H, \& Yudkin, J. (1972b). Proc. Nutr. Soc. 3I, 9A. 
Chevillard, L., Portet, R., Combescot, Ch. \& Senault-Bournique, S. (1968). (Quoted by Tremolières, J. \& Lavau, M. (1969). Cah. Nutr. Diet. 4, 83.)

Cohen, A. M., Teitelbaum, A., Balogh, M. \& Groen, J. J. (1966). Am. J. clin. Nutr. I9, 59.

Jackson, W. P. U., Mieghem, W. van \& Keller, P. (1972). Lancet i, ro40.

Mahler, R. (1 965). Q. $7 l$ Med. 34, 484 .

Miller, D. S. \& Mumford, P. (1967). Am. F. clin. Nutr. 20, 1212.

Perheentupa, I. \& Raivio, K. (1967). Lancet ii, 528 .

Stout, R. W. \& Vallance-Owen, J. (1969). Lancet i, 1078.

Szanto, S. \& Yudkin, J. (Ig69). Post-grad. med. 7. 45, 602.

Yudkin, J., Evans, E. \& Smith, M. G. M. (1972). Proc. Nutr, Soc. 31, 12A. 